\title{
Negotiating Effectively: Justice in International Environmental Negotiations
}

\author{
Cecilia Albin'1 . Daniel Druckman²,3,4
}

(C) The Author(s) 2016. This article is published with open access at Springerlink.com

\begin{abstract}
Are negotiators who rely on justice principles in the process of bargaining and drafting agreements more-or rather less-effective than others? This article examines whether adherence to principles of procedural and distributive justice in negotiations contributes to more effective results, with a focus on international environmental negotiations. Effectiveness is defined in terms of the extent of agreement (among parties and on issues), time to reach agreement, and comprehensiveness of the agreement. A set of hypotheses is evaluated on a selection of bilateral and multilateral cases of environmental negotiations, using statistical methods. The analyses reveal that adherence to principles of procedural justice contributes to more effective results in multilateral environmental negotiations. These principles are found to hinder effectiveness in the bilateral cases. On the other hand, adherence to principles of distributive justice is only moderately related to effectiveness in both the bilateral and multilateral cases.
\end{abstract}

Keywords Distributive justice $\cdot$ Environmental negotiations $\cdot$ Negotiation effectiveness $\cdot$ Procedural justice

$\otimes$ Cecilia Albin

Cecilia.Albin@pcr.uu.se

$\bowtie$ Daniel Druckman

dandruckman@yahoo.com

1 Uppsala University, Uppsala, Sweden

2 George Mason University, Fairfax, VA, USA

3 Macquarie University, Sydney, Australia

4 University of Queensland, Brisbane, Australia 


\section{Introduction}

Negotiators typically seek to reach effective agreements rather than costly compromises or extended impasses. How effective negotiations are brought about has been a matter of long-standing interest in the negotiation literature (e.g., Hopmann 1995; Jensen 1988; Sjöstedt 1993). Among the many factors explored are the role of different actors (e.g., third parties as mediators, non-governmental organizations), relations between negotiating parties (e.g., antagonistic, cooperative), the power balance (e.g., symmetric or asymmetric), and negotiation methods (e.g., integrative/problem solving vs. distributive/competitive). Less attention has been paid to the impact of justice considerations: Are negotiators who adhere to justice principles in the process of bargaining and drafting agreements more or less effective than others?

As international negotiations have become ever more important as a tool to tackle global issues, they have faced repeated stalemates or slowed down in a number of key areas. While the reasons are many and complex, justice issues are at the heart of the difficulties in many fields. For example, procedural issues regarding agenda-setting and fair party representation/inclusion have often become controversial stumbling blocks in negotiations within the World Trade Organization (WTO). From the outset the core of climate change negotiations has concerned distributive issues of justice over who should undertake greenhouse gas emission cuts (given inequalities in past, current and future projected emission levels), and at whose cost (given differences in responsibility for the problem, in resources and in gains to be had from emission abatement) (see Harris 2001, 2010). Earlier research has demonstrated that justice principles and concepts influence the dynamics of negotiation and the content of agreements (Coolsaet and Pitsays 2015; Hollander-Blumhoff and Tyler 2008; Zartman and Kremenyuk 2005).

Recent findings, specifically in the areas of trade and arms control, demonstrate important relationships between justice principles and the effectiveness of the negotiation (Albin and Druckman 2014a, b). The current study examines these relationships in another issue area, international environmental negotiations. This article systematically examines if and how procedural justice $(\mathrm{PJ})$ - that is, the justice of the process and procedures whereby negotiations are conducted — or distributive justice (DJ) the justice of the allocation of benefits and burdens in the outcome-impacts upon negotiation effectiveness.

We develop a set of hypotheses about the impact of PJ and DJ on the effectiveness of international environmental negotiations. As further discussed below, effectiveness is defined in terms of several dimensions, including the extent of agreement (among parties, on issues), time to reach agreement, and comprehensiveness. The hypotheses are evaluated on a selection of 20 cases of bilateral and multilateral environmental negotiations using statistical methods. The overarching purpose is to contribute to existing knowledge about factors in the process that contribute to the effectiveness of negotiations.

The focus on international environmental negotiations is chosen for several reasons. They represent a significant area of international activity: the protection of many environmental resources is now widely recognized as essential to national and international welfare, and as requiring successful trans-boundary cooperation. Yet negotiations in 
this area often suffer from slow progress and insufficiently effective results. They are characteristically marked by difficult questions of justice concerning obligations and cost-sharing because of inequalities between countries: inequalities in responsibility between polluting and victim countries, in sensitivity to environmental pollution and gains to be had from regulatory agreements, and in economic and technological ability to exploit valued resources as well as to implement protective measures (Coolsaet and Pitsays 2015). Negotiations over climate change, trans-boundary air pollution, ozone depletion, the protection of the world's seas and oceans, and international trade in toxic wastes, are only a few examples. There is no systematic comparative study of the impact of either PJ or DJ on negotiation effectiveness.

The first section outlines the study's approach to the question of how to define and recognize adherence to justice principles. Second, the most relevant research literature is reviewed and the hypotheses presented. Third, the data set and methods used are described. Fourth, the results of statistical analyses are presented. Fifth, implications of the findings are discussed. A concluding section presents broader, more far-reaching, implications of the findings.

\section{Approach}

This article examines how PJ and DJ principles impact upon effectiveness in environmental negotiations. It follows the mixed method approach developed for analyses of the relationship between justice and effectiveness in sets of bilateral and multilateral trade (Albin and Druckman 2014a) and arms control cases (Albin and Druckman 2014b). In each of these studies, we focus specifically on negotiation dynamics. This focus limits the time span to the bounded periods of the negotiation. We are aware however that talks occur within a broader context of policy formulation. For environmental negotiations this context includes debates over issues of inter-generational sustainability. We encourage further research that takes account of the longer time horizons in each of these areas.

Procedural justice refers to how the negotiations are conducted, how the parties relate to each other, and how they are treated during the process. Specifically, PJ is defined in terms of four principles (Lind and Tyler 1988). One is fair representation, referring to full or balanced representation in the decision-making process of parties and interests expected to be affected by the outcome. Another principle concerns issues of fair treatment and fair play, which refer to opportunities to have input, be heard and influence the process and consistency and impartiality in the conduct of it. A third is voluntary agreement, that is, freedom from imposition and acceptance of one's own volition. Finally, the principle of transparency refers to openness and accessibility regarding decision-making in the negotiation process, as parties work to reach a negotiated agreement.

Distributive justice refers to the justice of the allocation of benefits and burdens in the terms of agreements (Deutsch 1985). It is often discussed during the negotiation process and, when taken seriously by the parties, reflected in the outcome. DJ is defined in terms of four principles. The first is equality, referring to identical or comparable distributions of resources and burdens. The second is proportionality, implying distri- 
butions of resources and burdens in proportion to relevant inputs such as, for example, contributions made. A third is need, meaning distributions of resources to meet present basic needs. Finally, compensation denotes resource distributions to indemnify undue costs or wrongdoings inflicted upon a party.

Each of these eight principles is recognized as a central component of PJ and DJ, respectively, in the research literature even if its exact definition varies. They are highly relevant across issue areas including environmental negotiations, and lend themselves well to operational definition. In this study, DJ is examined not as an outcome but as a process variable - that is, how parties' notions of what DJ principles should guide the distributions of benefits and burdens in an agreement affected the process of negotiating.

The experimental and case-study literature on negotiation provides a foundation for our measures of effectiveness. An effectiveness index was constructed from the following five components:

- Number of issues resolved This is a frequently used outcome measure in a large variety of experimental studies (see Druckman's 1994 meta-analysis [80 experiments]) and comparative case studies (see Druckman et al. 1999 [30 cases]).

- Time to reach agreement This is a frequently used indicator of negotiation efficiency used particularly in experimental studies (see Druckman 1994). It can be regarded both as a feature of the process (duration of the negotiation) and of the outcome (anchored by the date of the agreement). It provides an indicator of the efficiency of the negotiation.

- Degree of agreement This indicator refers to the extent of support for an agreement among parties. In large-scale multilateral negotiations involving numerous parties and interests, agreements are often a challenge to achieve (see Albin and Druckman 2014a, b).

- Type of agreement This indicator refers to the comprehensiveness of the agreement, including the extent to which implementation issues are addressed. For example, an agreement may address all outstanding issues comprehensively or only partly, and may resolve them in depth or handle them more superficially. It was a key outcome measure in one comparative case study on negotiations to end international conflicts (Irmer and Druckman 2009 [26 cases] and in another on types of negotiating objectives (Druckman et al. 1999 [30 cases]).

- Balance of integrative and distributive elements This indicator captures Hopmann's distinction between absolute or joint and relative benefits (Hopmann 1995). It was a key measure of the quality of outcomes in a comparative case study of historical negotiations (Wagner 2008) and a core variable in a simulation experiment on procedural justice (Hollander-Blumhoff and Tyler 2008).

The five equally-weighted components form the index used in this study to evaluate the impacts of PJ and DJ. The index captures the effectiveness of the negotiation by including both process efficiency (time to reach agreement) and quality of agreement (issues resolved, degree and type of agreement) indicators. The three outcome indicators refer to the breadth and depth of cooperation achieved or enshrined in the agreement. Both the aggregated index and each of its five components are analyzed. 
An advantage of the approach is that it increases the relevance of a more general literature on negotiation for hypothesis development and evaluation.

In this comparative study we explored empirical relationships between two types of justice and the effectiveness of negotiation. Both the independent (justice) and dependent (effectiveness) variables were considered to be aspects of negotiation process (PJ, DJ, time to reach agreement) and features of its outcome (issues settled, type of settlement). These variables and the hypotheses that relate them to each other are drawn from the broad literature on negotiation and, more specifically, from the study of international negotiation. The findings contribute primarily to our understanding of negotiation with implications for environmental policy.

\section{Research Review and Hypotheses}

The research literature on international environmental negotiations explores a number of themes in depth, often with a focus on such talks at the multilateral level and through case studies. Yet despite this considerable body of literature and its importance in this domain, justice issues remain relatively little researched. Much of the literature that does focus on justice in environmental negotiations, and in environmental politics more generally, is concerned with the climate change problem and/or is normative, posing questions about how to conceptualize ethical obligations and the meaning of a just agreement or solution (Arnold 2011; Harris 2001, 2010; Gardiner et al. 2010; Müller 2001; Paterson 2001; Shue 1992). Following O'Neill, we agree that "any theory of justice that is to be taken seriously must respect empirical findings" $(2000,2)$. Yet, the empirical research literature on justice in the international context is limited with only a few studies examining how justice concepts and principles impact upon the dynamics of environmental negotiations (Lange et al. 2007, 2010; Albin 2001, Chapter 3). The overall focus is on distributive (outcome) justice rather than procedural (process) justice (e.g., Harris 2010). We address the fundamental question of how adherence (or not) to PJ and DJ principles in the negotiation process impacts upon effectiveness.

The importance of procedural justice principles is evident in a variety of international negotiations. Monheim's (2015) multilateral case comparisons are instructive. He showed that procedural issues were critical variables in whether an agreement was reached in each of three cases: the Copenhagen Climate Change Conference, the WTO trade negotiations in Doha following the failed Seattle round, and the Cartagena biosafety protocol negotiations. The shifts that occurred in each of these negotiations consisted of increased (or decreased) transparency and inclusiveness. Monheim's process tracing probes showed how decisions made by the organizers in the direction of openness (or limited transparency and fair representation) affected whether these negotiation processes moved from impasse to a path to agreement. More broadly, our recent comparative research on trade and arms control cases supports the connection between PJ and effectiveness. Strong statistical relationships were found for these variables in both the multilateral (10 cases) and bilateral (12 cases) trade negotiation samples (Albin and Druckman 2014a). A particularly strong relationship between 
adherence to PJ principles and effectiveness was demonstrated as well for the bilateral arms control cases sampled by Albin and Druckman (2014a).

With regard to large-scale multilateral negotiations, a focus on managing a complex process highlights the importance of PJ principles. This challenge has clear implications for transparency, fair representations, and fair treatment of all parties. It also has implications for voluntary agreements, especially when core deals are worked out by a small group and presented for approval to all of the other parties. Effective negotiations depend on implementing these principles. But, process management is only one benefit of adhering to PJ principles. Another benefit is relational. Agreement on PJ principles in negotiation has been shown to produce more problem-solving behavior which, in turn, lead to more integrative outcomes (Hollander-Blumhoff and Tyler 2008; Wagner and Druckman 2012). This path may be set in motion by the impact of PJ on trust. By enhancing perceived trust, the PJ principles create a negotiating climate that increases the prospects for durable agreements (Holtz 2013; Druckman and Wagner 2016).

These findings suggest a first hypothesis:

H1 If negotiators adhere to PJ principles during the process, then they will achieve more effective agreements. (PJ has a direct impact on effectiveness.)

It may also be the case that PJ hinders efficiency (time to reach agreement) and thwarts reaching agreements as Depledge (2005) notes in the context of global climate change negotiations. She observes that "excessive concern with procedural equity/transparency can jeopardize the reaching of agreement" (Depledge 2005, 232). Procedural rules may clash with interests. By encouraging equal participation and transparency, these rules reduce opportunities of a few powerful parties to influence decisions made by the many. In frustration, these more powerful parties may try to extend or subvert the negotiation resulting in impasses. Conversely, smaller countries may use the procedures to delay or otherwise subvert the negotiation in an effort to achieve their aims. She cautions that effective agreements depend on striking a balance between procedural equity and efficiency. Although this argument may apply specifically to large-scale global conferences, it can be considered as a basis for an alternative hypothesis as follows:

H1a If negotiators adhere to PJ principles during the process, then there will be less efficiency of the negotiation and less effective agreements. (PJ has a direct impact on effectiveness.)

Albin (2001) points to the importance of considering DJ principles in international negotiations, in order to reach effective agreements as defined in this study. It is logical to expect that when DJ is part of the process of negotiating the terms of agreements, the process will be more efficient and the outcome will be more effective. Particularly in negotiations in which significant burdens or valued resources are to be allocated, claims are unlikely to be successful unless they are based on some DJ principle (Elster 1992). Virtually all larger-scale multilateral negotiations at the international level, and many international bilateral negotiations, involve the allocation of major assets or costs. 
Another finding is that effective agreement often depends on accounting for and balancing several DJ principles in the negotiation process: a single principle can rarely take into account all relevant aspects in complex international cases (Albin 1995). More generally, Frohlich et al. (1987) found that the individuals in their laboratory experiments took into account more than one factor in the notion of DJ that guided their choices. They were rarely guided by a single-factor principle, preferring instead a mixed approach or a trade-off among different principles. This has also been demonstrated in international negotiations, including those on environmental issues (see, for example, Coolsaet and Pitsays 2015). Among many examples are the negotiations leading to the 1987 Montreal Protocol: They combined the principles of proportionality, compensatory justice, need and equality in drafting the agreement so as to take account of the varied conditions and concerns of participating states, particularly those of industrialized versus developing countries.

These findings suggest a second hypothesis:

H2 If DJ principles are adhered to during the negotiation process, then the negotiation will be more efficient and the agreements will be more effective. (DJ has a direct impact on effectiveness.)

The hypotheses above concern the independent and direct effects of PJ and DJ on outcomes. Another question is: How does PJ relate to DJ? One study that addresses this question holds that an outcome favoring one party more than another increases the importance of PJ while an outcome favoring all parties can help compensate for a lack of adherence to PJ principles during the process (Konovsky 2000). PJ and DJ have also shown to be strongly correlated across various contexts (Hauenstein et al. 2001) and the idea that more PJ in the process leads to more DJ in the outcome is supported (Brockner and Siegel 1996; Niehoff and Moorman 1993; Törnblom and Vermunt 2007).

These studies examine DJ as an outcome variable. However, the arguments and conclusions can be extended to hold also when DJ is a process variable, as in the present study. In other words, it is reasonable to expect that the more central PJ is in the negotiation process, the more central DJ will be in the process as well. The pursuit of just procedures is likely to enhance the willingness and opportunities to adhere to DJ principles as well when negotiating over and formulating the terms of an agreement. Thus, PJ may mediate the relationship between DJ and effectiveness. However, since both PJ and DJ are assessed during the process, a reverse causal direction is also plausible: DJ may mediate the relationship between PJ and effectiveness. Both directions, indicating indirect effects for these justice variables, are hypothesized as part of a third set of hypotheses:

H3 More adherence to PJ principles is associated with more adherence to DJ principles in the negotiation process. Conversely, less adherence to PJ principles is associated with less adherence to DJ principles in the negotiation process.

Indirect effects are suggested as corollaries to $\mathrm{H} 3$ :

H3a PJ mediates the relationship between DJ and effectiveness. (PJ has an indirect impact on effectiveness.) 
H3b DJ mediates the relationship between PJ and effectiveness. (DJ has an indirect impact on effectiveness.)

This set of hypotheses was evaluated across a sample of bilateral and multilateral environmental cases. The sampling provides an opportunity to use statistical methods as tools for verifying or falsifying each of the hypotheses. An advantage of this approach is that judgments about the hypotheses derive from analyses performed on a broad variety of cases. Emphasizing width more than depth, the analyses address issues of generality.

Performing statistical analyses entails a sequence of tasks. First, a sample of cases must be chosen according to pre-established selection criteria. Second, the justice and effectiveness concepts must be defined as scaled variables. Third, coders must be recruited and coding rules developed. Fourth, calibration procedures are needed to ascertain the reliability of the coders' judgments. Fifth, a final dataset must be assembled for the analyses. These tasks set the stage for the sixth task of performing statistical analyses. The Statistical Package for the Social Sciences (SPSS) was used to perform correlation and regression analyses designed to evaluate the hypotheses. These tasks are described in the sections to follow. They provide the methodological basis for the results and, thus, the value of the contribution to the literature on international environmental negotiations.

\section{Sampling Procedures}

Twenty cases of environmental negotiations, which have each resulted in an assessable outcome, were carefully selected to evaluate the hypotheses. Our aim was to choose cases that reflect the key dimensions of international environmental negotiations, including size, type of parties, types of issues being negotiated, time periods, and regions. This is a representative, rather than random, sampling of cases: Random sampling depends on a having access to a known universe or population of cases. ${ }^{1}$ These bilateral and multilateral cases are described in Supplemental Appendix A. ${ }^{2}$ With regard to the number of participating parties, ten of these were smaller-scale (bilateral, trilateral and quadripartite) and ten were large-scale (20 parties or more) so as to capture the different process dynamics involved. Although not large in a statistical sense, the number of cases chosen provides a good ratio of cases (20) to variables (3), thereby contributing to the power of the statistical tests used. Furthermore, the sample was chosen with a view to being representative of a larger population of such negotiations on four criteria.

One criterion is the type of party involved, with a focus on parties participating officially and/or directly in the talks. While state representatives still dominate,

\footnotetext{
1 The number of cases chosen was influenced as well by the availability of resources for process and outcome coding. These are labor-intensive tasks that include finding sufficient documentation about each case and entail time-consuming calibration procedures discussed in the next section. We aimed to achieve a balance among variety, documentation, coding resources, and a sufficient number of cases for performing statistical analyses.

${ }^{2}$ Supplemental Appendix A, consisting of information about each case, is available from the authors.
} 
environmental negotiations often include other types of actors. As the sample in Supplementary Appendix A illustrates, this is especially relevant to large-scale environmental talks: The complex issues with which they deal and the relatively open public fora in which they take place require or invite several types of actors. Another criterion consists of the key issues negotiated. The sample is representative of the great variety of issues covered in environmental negotiations and includes climate change, ozone depletion, air and nuclear pollution, fisheries, waterways, hazardous wastes and biodiversity.

Third, cases were chosen with a view to include different time periods: In this sample they range from the earliest case concluded in 1911 and the most recent in 2009. The time required to conclude the official talks also differs widely, from many years in several cases to 13 days (the 2009 Copenhagen climate change conference). A final criterion of selection concerns the regions (for small-scale cases) and fora (for large-scale cases, which typically transcend regional boundaries) in which the negotiations took place. Various divisions within the United Nations system predominate among the fora, in the sample and in the larger population of cases. While several regions are included, North America is somewhat overrepresented due to the greater difficulty of securing sufficiently detailed documentation on the negotiation process in other regions. Overall, the cases provide a reasonably representative sample of environmental negotiations. The variety of cases provides a robust sampling that increases the chances for generality to other cases in this area of negotiation (Druckman 1994).

Another concern with regard to the sampling process is the possibility of a selection bias. This refers to biases that increase the chances of confirming the research hypotheses, or to selecting on the dependent variable, which in the present study is effectiveness. For example, the sample could reflect a limited range of effectiveness ratings or target cases where the justice variables correlate with effectiveness. Precautions were taken to prevent these biases from occurring. One precaution consisted of choosing the cases before operationalizing the effectiveness variable. Another was to develop the research hypotheses after the sample was chosen. Further, the coders were blind to the research hypotheses, which were developed by the authors. These procedural decisions enhance our confidence in judging that we neither selected the cases on the dependent variable nor on the justice-effectiveness hypotheses discussed in the previous section.

\section{Variables, Coding and Analysis Methods}

Following the procedures used in the Albin and Druckman (2014a,b) studies, three variables were analyzed in each of the 20 cases: PJ and DJ principles in the process, and the effectiveness of the agreement. Since DJ is about the distribution of benefits and burdens, it is usually assessed in the terms of agreements. Because the dependent variable, effectiveness, is also about the outcome, it would have been difficult to distinguish it from assessments of DJ in agreements. Procedures were therefore developed for assessing the adherence to DJ principles in the bargaining process. In other words, the analysis focused on whether and how parties' notions of DJ principles (if any) ought to underlie an agreement: For example, conflicting ideas of what 
DJ principle(s) should guide the distribution of benefits and costs in an agreement are likely to cause stalemate in negotiations, while similar ideas tend to facilitate the process.

A first step was to recruit two experienced coders. One coder was a professional expert on environmental negotiation with knowledge of a wide range of historical bilateral and multilateral cases. Another coder was a graduate student at Uppsala University with in depth experience in coding justice and effectiveness variables in the domains of trade and arms control. Neither was aware of the hypotheses being evaluated. A second step consisted of amassing sources on the negotiation process for each of the 20 cases. The documentation came primarily from secondary sources including both journal articles and books that described the negotiation process. ${ }^{3}$ These sources were augmented for a number of cases with reflections of participants in the negotiations. Guidebooks for coding the justice and effectiveness variables are provided in Supplemental Appendix B. These guides provide definitions for each of the variables and an example of application, the Canada-Spain Turbo negotiation. ${ }^{4}$ In order to avoid a possible order effect, the justice and effectiveness variables were coded in randomly-determined orders. For some cases justice coding preceded effectiveness, for other cases the order was reversed. Order alternation was also used for the two justice variables, PJ and DJ.

A next step for the coders was to identify procedural and distributive issues that arose in the negotiation process. The questions asked were: What kind of influence did the DJ (PJ) principle have with regard to those issues (primarily positive, mixed, or primarily negative)? How much of an impact did the principle(s) have on the negotiation process (highly significant, important, marginal)? For example, in the negotiations leading up to the 1987 Montreal Protocol, numerous procedural issues arose about integrating different parties' interests and input in the process, and about the tactics used by the European Community to stall the talks. In resolving these, all four PJ principles covered in this study turned out to play an important role.

Numerous distributive issues arose as well, mostly concerned with who should undertake what level of CFC emission cuts and against what baseline year, and with special provisions for developing countries. Unusually, all DJ principles included in this study were found to be at play. In the negotiation process, these principles had a highly significant impact, which was either positive or mixed.

Answers to questions for all the cases were combined to form an index that ranged from positive, highly significant (3) to negative, highly significant (0) in increments of .5 , resulting in a seven-step scale. This scale was designed to capture the overall impact of each principle identified in the process. Coding examples are provided in Supplemental Appendix B. A per principle average was also calculated by dividing

\footnotetext{
${ }^{3}$ A focus on process is not limited to statements made by particular negotiators. The descriptions may reflect rules of procedure or distributions, unwritten practices, chair persons, bureaus or, for large-scale conferences, the secretariat. We were interested in how the process played out with regard to the justice principles. For more on the organization of environmental negotiations see Depledge (2005) and Monheim (2015). A focus on sources for statements made during the process would be a useful complement to our work.

${ }^{4}$ Supplemental Appendix B, consisting of coding guides for procedural justice, distributive justice, and effectiveness, is available from the authors.
} 
the total score by the number of DJ or PJ principles coded. For example, a total score of 1.5 calculated from two principles results in a per principle score of .75 .

A three-step procedure was designed to calibrate the judgments of PJ and DJ made by the coders. These steps consisted of: (a) confronting differences among the coders, (b) reconciling those differences through discussion and, (c) arbitrating any nonreconciled coder differences by the authors. These procedures produced agreement on all but a few judgments: The agreement ratios were similar to those obtained in the earlier studies on trade and arms control negotiations, ranging from 78-86\% agreement depending on the type of negotiation (bilateral or multilateral) and type of justice principle (PJ or DJ).

Each calibrated effectiveness score was accompanied by a confidence judgment, consisting of a choice among fully, somewhat, not confident. The five coded part scores were aggregated for two indices, total and corrected effectiveness. The latter was corrected by the confidence ratings with weights of 1 (fully), .75 (somewhat), and .5 (not confident). The corrected index was used in the analyses reported below, with scores ranging from 0 (minimum corrected effectiveness) to 20 (maximum corrected effectiveness).

The hypotheses were evaluated with statistical procedures. The direct relationships between the justice variables (PJ and DJ) and effectiveness (Hypotheses 1,2) were assessed with correlational techniques. In order to control for effects of the other justice variable (either DJ or PJ), partial correlations were computed: For example, the PJ-effectiveness correlation was evaluated without variance contributed by DJ to PJ or effectiveness. Factor analyses were also performed on the correlations. This data reduction technique demonstrated clusters of closely related variables: For example, the PJ indices (total and per principle) and effectiveness.

The indirect relationships posited by the third set of hypotheses were evaluated with regression-based procedures. Referred to as mediation analysis (see Baron and Kenny 1986), these procedures indicate the extent to which DJ (or PJ) accounts for the PJ (or DJ)-effectiveness relationship.

\section{Results}

The tasks discussed in the sections on "Sampling Procedures" and "Variables, Coding, and Analysis Methods" produced the data sets shown in Tables 1 and 2. The calibrated scores for each coded variable are shown for the bilateral/small multilateral cases in Table 1. Table 2 presents the calibrated scores for the large multilateral cases. A first set of analyses was performed on the twenty combined bilateral and multilateral cases samples. The obtained correlations among the variables are shown in Table 3. A second set was performed on the bilateral/small-scale multilateral cases. The largescale multilateral cases were the basis for a third set of analyses. ${ }^{5}$

We begin with an evaluation of the competing hypotheses about the relationship between PJ and effectiveness.

\footnotetext{
5 Disaggregated data for the five components of the corrected effectiveness index are available from the authors.
} 
Table 1 Variables and codes for bilateral and small-scale multilateral cases

\begin{tabular}{llllll}
\hline Case & DJ total & DJ per & PJ total & PJ per & $\begin{array}{l}\text { Corrected } \\
\text { effectiveness }\end{array}$ \\
\hline US-Canada Air Quality & 5.5 & 2.75 & 7 & 2.33 & 17.25 \\
Canada-Spain Turbot Agreement & 8 & 2.6 & 5.5 & 1.6 & 18 \\
Pacific Salmon & 6 & 3 & 10 & 2.5 & 14.25 \\
Sealing Convention & 11 & 2.75 & 8.6 & 2.83 & 15.5 \\
Water Utilization & 8.25 & 2.75 & 9.5 & 2.38 & 15.5 \\
Jordan-Israel Water & 7 & 1.75 & 7.83 & 2.61 & 15.25 \\
Mekong Agreement & 1.75 & .44 & 7.25 & 2.42 & 13.25 \\
La Plata River & 4.88 & 2.44 & 10 & 2.5 & 14.25 \\
NAFTA Environmental & 5.66 & 2.8 & 6.5 & 1.62 & 15.25 \\
US-Singapore Free Trade Environmental & 4.5 & 2.25 & 6 & 3 & 14.75 \\
\hline
\end{tabular}

Table 2 Variables and codes for large-scale multilateral cases

\begin{tabular}{|c|c|c|c|c|c|}
\hline Case & DJ total & DJ per & PJ total & PJ per & $\begin{array}{l}\text { Corrected } \\
\text { effectiveness }\end{array}$ \\
\hline $\begin{array}{l}\text { Convention on assistance in the case of a } \\
\text { nuclear accident or radiological } \\
\text { emergency }\end{array}$ & 7.83 & 1.96 & 7.25 & 2.42 & 15.25 \\
\hline Convention on biological diversity & 6.25 & 1.56 & 7.13 & 1.78 & 12.00 \\
\hline Copenhagen climate change conference & 6.60 & 1.65 & 3.25 & .81 & 7.50 \\
\hline $\begin{array}{l}\text { Convention on the Regulation of } \\
\text { Antarctic Mineral Resource Activities }\end{array}$ & 3.87 & 1.29 & 7.25 & 2.42 & 12.75 \\
\hline $\begin{array}{l}\text { Convention on the Control of } \\
\text { Transboundary Movements of } \\
\text { Hazardous Wastes and their Disposal }\end{array}$ & 4.50 & 1.50 & 4.25 & 1.42 & 11.25 \\
\hline $\begin{array}{l}\text { Montreal Protocol on Substances that } \\
\text { Deplete the Ozone Layer }\end{array}$ & 8.21 & 2.05 & 7.67 & 1.92 & 15.25 \\
\hline $\begin{array}{l}\text { Stockholm Convention on Persistent } \\
\text { Organic Pollutants }\end{array}$ & 5.88 & 1.96 & 8.96 & 2.24 & 16.00 \\
\hline $\begin{array}{l}\text { UN Convention on the Law of Sea } \\
\text { (UNCLOS) II }\end{array}$ & 6.00 & 1.50 & 3.58 & 1.19 & 7.00 \\
\hline Convention on Watercourses & 6.58 & 1.65 & 7.80 & 1.95 & 12.50 \\
\hline $\begin{array}{l}\text { International Whaling Commission } \\
\text { Whaling Moratorium }\end{array}$ & 3.67 & 1.22 & 8.50 & 2.13 & 11.00 \\
\hline
\end{tabular}

Table 3 Correlations among the variables for the total sample (bilateral and multilateral cases)

\begin{tabular}{|c|c|c|c|c|c|}
\hline & DJ total & DJ per & PJ total & PJ per & Effectiveness \\
\hline DJ total & & $.59 * *$ & .11 & .01 & .31 \\
\hline DJ per & & & .32 & .22 & $.58 * *$ \\
\hline PJ total & & & & $.72 * *$ & $.54 * *$ \\
\hline PJ per & & & & & .60 ** \\
\hline
\end{tabular}

** Correlation is significant at the .01 level 
H1 If negotiators adhere to PJ principles during the process, then they will achieve more effective agreements. (PJ has a direct impact on effectiveness.)

H1a If negotiators adhere to PJ principles during the process, then there will be less efficiency of the negotiation and less effective agreements. (PJ has a direct impact on effectiveness.)

Analyses of the combined cases showed significant bi-variate correlations PJ total and effectiveness $(.54, p<.01)$ and PJ per principle and effectiveness $(.60, p<.003)$. These correlations change very little when controlling for the DJ variables. Factor analysis results show that the PJ and effectiveness variables cluster together on the same factor. These results support Hypothesis 1 but not Hypothesis 1a: PJ has a direct impact on effectiveness across the cases.

Analyses of the separate sub-samples show that the combined cases results are accounted for largely by the large-scale multilateral cases. For the bilateral/small multilateral cases, neither PJ variable (total or per principle) correlates significantly with effectiveness. However, a significant partial negative correlation is obtained $(-.67, p<.05)$ between PJ total and effectiveness when controlling for DJ total: More adherence to PJ in these cases leads to less effective negotiations particularly with regard to the balance component of the effectiveness index. In contrast, for the multilateral cases, both PJ variables show very strong relationships with effectiveness: Correlations of .81 $(p<.005)$ and .82 $(p<.004)$ for PJ total and PJ per respectively. The correlations are even stronger when controlling for DJ. Similarly, very strong regression coefficients (at $p<.004$ for PJ per principle and $p<.006$ for PJ total) indicate effectiveness is predicted by PJ. This relationship is illustrated for PJ per principle in Fig. 1: High PJ/high effectiveness cases are distinguished clearly from those that are medium $\mathrm{PJ} /$ medium effectiveness and low $\mathrm{PJ} /$ low effectiveness. These results are bolstered further by the factor analysis, where the PJ variables and effectiveness cluster together on the same factor. Thus, strong direct effects of PJ on effectiveness occur for the multilateral but not the bilateral cases.

The relationship for the multilateral cases is strong as well for three of the five components of the effectiveness index: number of issues resolved (.85 for PJ total, 76 for PJ per); degree of agreement $(.61, .69)$; type of agreement $(.75, .73)$. Somewhat weaker correlations occur for time to agreement and balance of the agreement. Thus, adherence to PJ principles during the negotiation process improves the quality of the agreements (number issues resolved, degree and type of agreement) but not the efficiency (time) of the process.

A factor analysis shows a cluster that includes both the effectiveness components and the PJ variables. Interestingly, the DJ variables are not part of this cluster, These findings provide support for Hypothesis 1 with regard to the multilateral cases but support Hypothesis 1a for the bilateral cases: PJ improves the effectiveness of the multilateral negotiations while diminishing the effectiveness of the bilateral cases.

Further analyses were performed on the four types of PJ. The strongest correlation was obtained between fair representation and effectiveness $(\mathrm{r}=.79)$. Representation predicted effectiveness $(\beta=.79 ; \mathrm{t}=3.66, p<006)$. Strong correlations were obtained between fair representation and three of the five components of effectiveness 


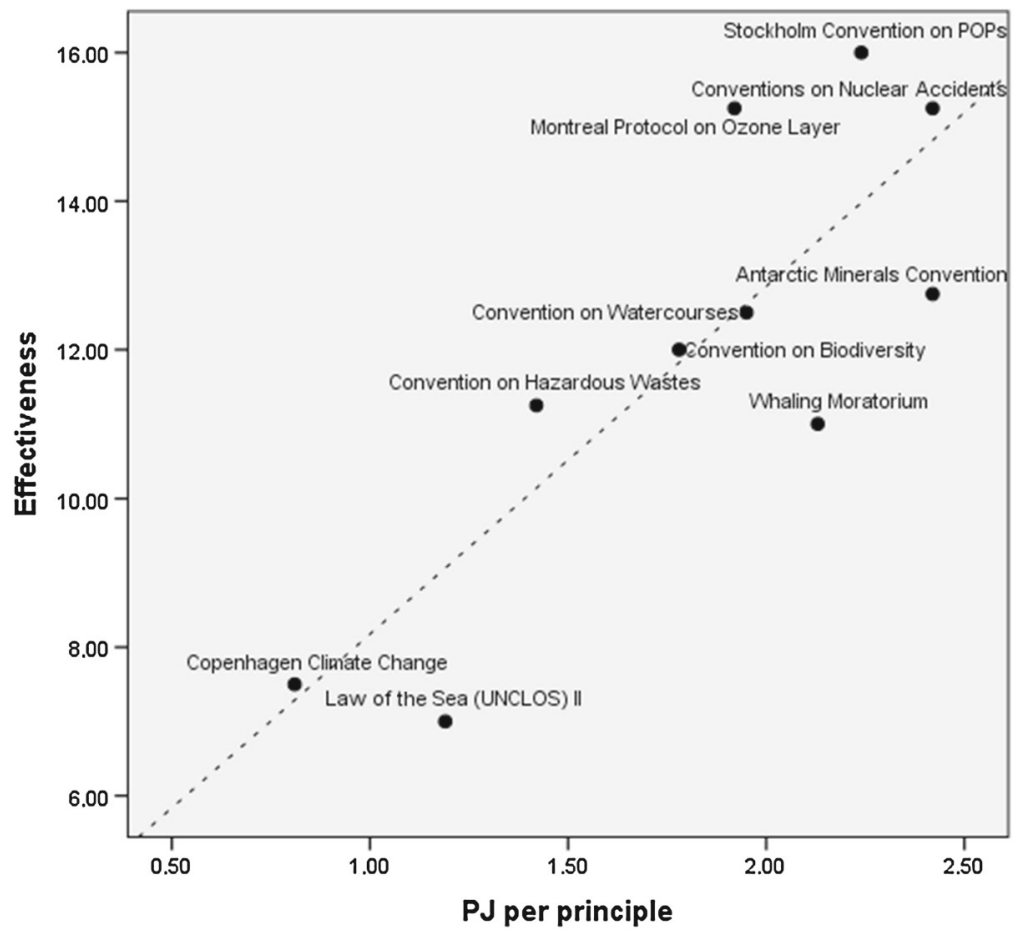

Fig. 1 Procedural justice by effectiveness for the multilateral cases

(number issues resolved [.79, $p<.008]$; degree of agreement [.82, $p<.004]$, and time to reach agreement $[.63, p<.05]$. More adherence to the principle of fair representation led to more agreement and more support for those agreements in a shorter period of time than less adherence to this principle. The relationship between voluntary agreements and effectiveness approached significance $(\mathrm{r}=.56)$. This $\mathrm{PJ}$ principle was a moderate predictor of effectiveness $(\beta=.56 ; \mathrm{t}=1.90, p<.09)$. A strong correlation was obtained between voluntary agreements and number of issues resolved $(\mathrm{r}=.79, p<.007)$ : More voluntary agreements led to the resolutions of more issues in the multilateral cases. These two types of PJ are also strongly correlated $(\mathrm{r}=.70, p<.02)$. This is due to the strong relationships to number of issues resolved. Partial correlations between representation and issues controlling for voluntary agreements and between voluntary agreements and issues controlling for representation decrease substantially: from .78 to .52 and from .79 to .54 respectively.

H2 If DJ principles are adhered to during the negotiation process, then the negotiation will be more efficient and the agreements will be more effective. (DJ has a direct impact on effectiveness.)

Analyses of the combined cases showed a significant relationship between DJ per principle and effectiveness $(.58, p<.007)$. The correlation is about the same when controlling for PJ per principle. The DJ effects are strong for both the bilateral and multilateral cases. 
For the bilateral cases, only moderate correlations occur between DJ total (.53, $p<.11)$ and DJ per principle $(.50, p<.14)$ and effectiveness. The pattern of correlations is similar for the components of the effectiveness index with near significant correlations with DJ for number of issues (.62 for DJ total) and the time measure (.58 for total and .54 for DJ per). However, DJ total correlates strongly with effectiveness when controlling for PJ total $(.71, p<.04)$ with strong partial correlations for the number of issues resolved (.61), time (.76), and type of agreement components (.51). This relationship is bolstered further by the factor analysis results: Strong clusters were found for the DJ and effectiveness variables; the PJ variables were not part of this cluster. These findings provide moderate support for Hypothesis 2.

It is also interesting to note that the effectiveness index is driven largely by the time to agreement (efficiency) component for the bilateral cases. This component has the strongest correlations with the two DJ measures and with the overall effectiveness index (.70). Further, factor analysis results show that this measure clusters with the two DJ variables. Thus, efficiency is the key aspect of effective negotiations for the bilateral cases. These cases are more efficient when the negotiating parties adhere to DJ principles during the process.

For the multilateral cases, DJ per principle correlates moderately with effectiveness $(.59, p<.07)$. A similar pattern occurs for the components of the index with nearsignificant correlations between DJ per and the time and balance measures. The DJ per variable correlates strongly with effectiveness when controlling for PJ per principle $(.86, p<.003)$; the partial correlations are particularly strong for the time, type of agreement and balance components of the index. The correlation between DJ total and effectiveness is not significant (.29). Factor analysis results show that DJ per principle (but not DJ total) clusters with effectiveness. But, the DJ variables also cluster on a second factor that does not include effectiveness. These results provide moderate support for the second hypothesis.

H3 More adherence to PJ principles is associated with more adherence to DJ principles in the negotiation process. Conversely, less adherence to PJ is associated with less adherence to DJ in the negotiation process.

H3a PJ mediates the relationship between DJ and effectiveness. (PJ has an indirect impact on effectiveness.)

H3b DJ mediates the relationship between PJ and effectiveness. (DJ has an indirect impact on effectiveness.)

None of the correlations between the PJ and DJ variables approaches significance for the combined or separate samples of cases. The highest correlations among the 12 relationships (various combinations of the PJ and DJ variables) is .32 (PJ total with DJ per principle). Thus, the PJ and DJ variables have independent effects on effectiveness, indicating a lack of support for $\mathrm{H} 3$.

The next question asks whether PJ accounts for the relationship between DJ and effectiveness. Results of mediation analyses for the combined and separate samples showed no significant role for PJ; nor was the DJ variable shown to mediate the relationship between PJ and effectiveness. Thus, both PJ and DJ had only direct impacts 
on effectiveness. The indirect effects Hypotheses $3 \mathrm{a}$ and $3 \mathrm{~b}$ are not supported by these analyses.

In summary, the statistical analyses showed the following:

Hypothesis 1 PJ has a strong direct effect on negotiating effectiveness. The effect is stronger for large-scale multilateral than for bilateral or small multilateral environmental cases. Adherence to these principles during the multilateral negotiation process improves the quality of the agreements (number issues resolved, degree and type of agreement) but not the efficiency (time) of the process. Fair representation was the type of PJ principle that correlated most strongly with effectiveness including three of the five components of this variable. Voluntary agreements correlated strongly with the number of issues resolved.

Hypothesis 2 DJ has a moderately strong direct effect on negotiating effectiveness for both the bilateral and multilateral cases. The key indicator of effectiveness in the bilateral cases is time to reach agreement. These cases are more efficient in terms of time spent negotiating when the parties adhere to DJ principles during the process.

Hypothesis 3 The PJ and DJ variables have independent effects on outcomes. These variables are not significantly correlated.

Hypothesis 3a PJ does not mediate the relationship between DJ and effectiveness. PJ effects are direct.

Hypothesis 3b DJ does not mediate the relationship between PJ and effectiveness. DJ effects are direct.

\section{Discussion}

The strongest effect occurs for the relationship between PJ and effectiveness in the multilateral cases. This effect is particularly strong for the number of issues resolved, degree and type (or comprehensiveness) of the agreement components of the index. Thus, PJ principles impact more on the quality of the multilateral agreement than the efficiency of the process. Regarding internal validity, the argument for causality is supported by the time lag between justice during the process and these components of effectiveness of the outcome. With regard to external validity, we extend the range of multilateral negotiations examined, providing evidence for the generality of Monheim's (2015) process tracing findings on the climate change, WTO trade, and biosafety negotiations.

Of interest are possible explanations for this finding. One explanation turns on normative arguments. Similar to trade talks, multilateral environmental negotiations are conducted in a normative setting where justice-related procedures influence rules of conduct. Process justice is often regarded as important: More powerful countries may be willing to settle for less in an agreement (Kapstein 2006). Another reason is that PJ principles are used by some actors to enhance trust or the perception of trustworthiness. The enhanced trust then increases the chances for obtaining effective agreements. A third is based on forward-looking effects of just behavior during 
the negotiation. Adhering to PJ principles increase confidence in the ratification and implementation of multilateral agreements as well as relationships among the parties. Thus, three mechanisms are suggested: belief in the importance of PJ, enhanced trust among the parties, and confidence going forward. These psychological effects may be instrumental in facilitating the coordination needed to move the large number of parties toward agreement. They may be set in motion by effective leaders as discussed by Karlsson et al. (2011) and demonstrated by Monheim (2015). The contrasting effects found for PJ in the bilateral and multilateral samples may reflect differences in the challenges of organizing the process and developing trusting relationships in which the delegates appreciate their shared identities.

The important role played by fair representation is an interesting finding. This PJ principle had the largest impact on effectiveness and its component parts. In his treatment of effective practices in multilateral negotiations, Monheim (2015) emphasizes the importance of transparency and inclusiveness. For him, these two aspects of PJ go hand in hand: an inclusive process includes openness in the broad transmission of information. In this study, we separated these features of the process and found that inclusiveness had a stronger impact on effectiveness than transparency in our multilateral cases. Nor were these two features correlated. Fair representation was strongly related to voluntary agreements. Thus, inclusion, voice and a lack of coercion were the hallmarks of effective multilateral negotiations in the sampled cases. These findings have theoretical implications by raising the question of whether these aspects of PJ increase perceptions of trust and shared identities among the delegates more than transparency and fair play. But the findings also have practical implications by identifying areas where organizers can apply their leverage on the process most effectively.

Further understanding of the PJ-effectiveness relationship is provided by taking a closer look at some of the cases in our multilateral sample. They provide a ground-eye view of the justice-effectiveness relationship. An interesting contrast is between the way that adherence to PJ principles contributed to effectiveness in the Convention on Nuclear Accidents case with the way that less adherence to those principles hindered effectiveness in the Basel Convention on Hazardous Wastes case. The Convention on Nuclear Accidents, negotiated during July and August 1986, is an example of a high $\mathrm{PJ} /$ high effectiveness case. Implementation of key PJ principles paved the way to the final agreement. Actions taken by the IAEA and the respective chairs of three working groups assured fair treatment for all the parties. Serving as an impartial facilitating organization, the IAEA prepared a draft text, provided expert services, and encouraged a continuous flow of information. The chairs of the working groups developed strategies that encouraged exchanges of views and dispute resolution procedures for dealing with difficult issues. They also assured broad participation and voluntary decisions, adding to the legitimacy of the emerging consensus among the parties. A constructive process combined with efficiency to produce a consensual and timely agreement, contributing to the high effectiveness score.

The Basel Convention on Hazardous Wastes, negotiated from February 1988 to March 1989, presents a different picture. Moderate adherence to some principles led to moderately effective agreements. A lack of transparent decisions was balanced by a consensus rule for adoption of the convention, thereby giving the appearance of 
voluntary decision making. A lack of country representation on sub-committees was balanced by open participation for all member states of the United Nations. However, domination by the industrialized countries may have created an impression of false justice, whereby procedural principles were used to secure favorable agreements.

The importance of getting an agreement trumped the status quo as an alternative for many parties. This is reflected in several parts of the effectiveness index: less comprehensive, vague, and more distributive than integrative. A lack of viable alternatives for many parties and an efficient process led to somewhat higher codes for degree of agreement (partial support from all parties) and time to reach agreement (only minor delays).

It is also interesting to note that $\mathrm{PJ}$ is difficult to achieve in large complex cases. The lowest PJ and effectiveness scores shown in Table 2 occur for the climate change and Law of the Seas cases. These were also the largest negotiating forums in the multilateral sample. Adherence to PJ principles in these large conferences might entail giving (too) much free play and room for conflicting interests and parties to reduce the possibilities of an effective agreement.

The inverse relationship between PJ and effectiveness in the bilateral cases-in particular, the parts on integrative/distributive balance and time-is harder to explain. The reason may have to do with bargaining power: While it is typically dispersed in multilateral environmental negotiations, it is often unequally distributed between polluting states and states victimized by pollution when they negotiate in bilateral venues. The introduction of PJ in the latter case may then create an artificial kind of procedural equality which collides with the reality of unequal bargaining parties, thereby hindering effective negotiation. It is well known that equality of power between negotiating parties facilitates an integrative bargaining approach (Wagner 2008).

With regard to the moderately strong DJ-effectiveness relationships, it is interesting to note that adhering to DJ principles primarily improved the effectiveness of the bilateral negotiation cases. Proportional or equal distribution decisions moved the talks more quickly toward agreement. Since these are also features of the negotiated outcome, it would seem plausible that progress on these matters made during the process would facilitate movement down the path to agreement.

It is the case that we focused selectively on justice variables. No doubt other factors were at play in producing effective (or ineffective) outcomes and shifts in environmental policy by the countries represented (see, for example, Depledge 2005; Monheim 2015). Our decision to limit the focus of this study to a few theory-relevant variables allows us to evaluate the impact of these variables across a wide array of different cases. Robust findings are an advantage of case variety, contributing to confidence about generalizing the findings to other settings. But, we are also aware of the need to embed the justice variables in broader frameworks of factors that influence negotiation effectiveness.

One aspect of the broader context is the sequential or temporal feature of many environmental negotiations (Downie 2013). For example, the outcome of the Copenhagen Climate Change Conference was influenced by negotiations that took place over two years. More generally, agreements can be considered to be part of a policy formulation process that is followed by implementation problems not foreseen by the negotiators (see Bumpus and Liverman 2011; Okereke and Dooley 2010; Okereke and Schroeder 
2009; Spector and Zartman 2003). Extending the time line used for analysis also invites consideration of issues concerned with the sustainability of agreements, particularly the debates surrounding relationships between DJ and environmental sustainability. Although both justice and sustainability are central goals of negotiated agreements, they may also be conflicting goals as Dobson (1998) has pointed out. Neither equal nor equitable outcomes guarantee inter-generational environmental integrity. These debates have important implications for how effectiveness is conceived and deserve serious attention in further research on justice in environmental negotiations.

\section{Conclusion}

The overriding conclusion of this research is that justice matters in international environmental negotiations. Justice issues are not only raised and argued about, but actually influence the direction of the process and the eventual outcome. These findings provide a balance to analyses that focus primarily on interests and power while largely ignoring justice and related moral/ethical norms. Interestingly, this balance is particularly germane to the field of international relations (Muller and Druckman 2014). A plethora of studies in cognate social science fields, many reviewed in this article, supports the central role played by justice. Extending this support to the international domain is an important contribution of our work. But, the work goes further by distinguishing between types of justice and the scale of the negotiations.

The key finding is the strong PJ-effectiveness relationship in the multilateral cases. The relationship is particularly strong for the "quality of agreement" (issues resolved, degree and type of agreement) components than for the efficiency (time to agreement) components of effectiveness. A key to this relationship is adherence to the principle of fair representation. Thus, in accordance with Monheim's analysis, PJ leads to better environmental agreements. The practical implications of these findings are captured well by Monheim's (2015) seven prescriptions dealing with information and inclusiveness. The DJ-effectiveness findings are also interesting. Although the overall relationships between these variables are moderate, the components analyses reveal that the time measure relates strongly to DJ for the bilateral cases. Thus, raising DJ issues during the process encourages a more efficient negotiation.

Acknowledgements The authors gratefully acknowledge support from Riksbankens Jubileumsfond, Sweden (Grant No. P09-0448:1-E) for the research reported in this article. Considerable gratitude goes to Lynn Wagner and Annkatrin Tritschoks for their many helpful contributions to the study.

Open Access This article is distributed under the terms of the Creative Commons Attribution 4.0 International License (http://creativecommons.org/licenses/by/4.0/), which permits unrestricted use, distribution, and reproduction in any medium, provided you give appropriate credit to the original author(s) and the source, provide a link to the Creative Commons license, and indicate if changes were made.

\section{References}

Albin C (1995) Rethinking justice and fairness: the case of acid rain emission reductions. Rev Int Stud 21(2):119-143 
Albin C (2001) Justice and fairness in international negotiation. Cambridge University Press, Cambridge

Albin C, Druckman D (2014a) Procedures matter: justice and effectiveness in international trade negotiations. Eur J Int Relat 20(4):1014-1042

Albin C, Druckman D (2014b) Bargaining over weapons: justice and effectiveness in arms control negotiations. Int Negot 19(3):426-458

Arnold DG (ed) (2011) The ethics of global climate change. Cambridge University Press, Cambridge

Baron RM, Kenny DA (1986) The moderator-mediator variable distinction in social-psychological research: conceptual, strategic, and statistical considerations. J Pers Soc Psychol 51(6):1173-1182

Brockner J, Siegel PA (1996) Understanding the interaction between procedural and distributive justice: the role of trust. In: Kramer R, Tyler T (eds) Trust in organizations: frontiers of theory and research. Sage, Thousand Oaks

Bumpus AG, Liverman DM (2011) Carbon colonialism? Offsets, greenhouse gas reductions and sustainable development. In: Peet R, Robbins P, Watts M (eds) Global political ecology. Routledge, Abingdon

Coolsaet B, Pitsays J (2015) Fair and equitable negotiations? African influence and the international access and benefit-sharing regimes. Glob Environ Polit 15(2):38-56

Depledge J (2005) The organization of global negotiations: constructing the climate change regime. Earthscan, London

Deutsch M (1985) Distributive justice: a social-psychological perspective. Yale University Press, New Haven

Dobson ANH (1998) Social justice and the environment: conceptions of environmental sustainability and dimensions of justice. Oxford University Press, New York

Downie C (2013) Three ways to understand actors in international negotiations: Climate change in the Clinton years (1993-2000). Glob Environ Polit 13(4):22-40

Druckman D (1994) Determinants of compromising behavior in negotiation: a meta-analysis. J Confl Resolut 38(3):507-556

Druckman D, Wagner L (2016) Justice and negotiation. Annu Rev Psychol 67:387-413

Druckman D, Martin J, Nan SA, Yagcioglu D (1999) Dimensions of international negotiation: a test of Ikle's typology. Group Decis Negot 8(2):89-108

Elster J (1992) Local justice: how institutions allocate scarce goods and necessary burdens. Russell Sage Foundation, New York

Frohlich N, Oppenheimer JA, Eavey CJ (1987) Laboratory results on Rawls's distributive justice. Br J Polit Sci 17(1):1-21

Gardiner S, Caney SM, Jamieson DM, Shue H (eds) (2010) Climate ethics: essential readings. Oxford University Press, Oxford

Harris PG (2001) International equity and global environmental politics: power and principles in U.S. foreign policy. Ashgate, Aldershot

Harris PG (2010) World ethics and climate change: from international to global justice. Edinburgh University Press, Edinburgh

Hauenstein NMA, McGonigle T, Flinder SW (2001) A meta-analysis of the relationship between procedural justice and distributive justice: implications for justice research. Empl Responsib Rights J 13(1): 39-56

Hollander-Blumhoff R, Tyler T (2008) Procedural justice in negotiation: procedural fairness, outcome acceptance, and integrative potential. Law Soc Inq 33:473-500

Holtz BC (2013) Trust primacy: a model of the reciprocal relations between trust and perceived justice. J Manag 39(7):1891-1923

Hopmann PT (1995) Two paradigms of negotiation: bargaining and problem solving. Ann Am Acad Polit Soc Sci 542:24-47

Irmer C, Druckman D (2009) Explaining negotiation outcomes: process or context? Negot Confl Manag Res 2(3):209-235

Jensen L (1988) Bargaining for national security: the postwar disarmament negotiations. University of South Carolina Press, Columbia

Kapstein E (2006) Economic justice in an unfair world: towards a level playing field. Princeton University Press, Princeton

Karlsson C, Parker C, Hjerpe M, Linner B-O (2011) The legitimacy of leadership in international climate change negotiations. Glob Environ Polit 11(1):89-107

Konovsky M (2000) Understanding procedural justice and its impact on business organizations. J Manag 26(3):489-511 
Lange A, Vogt C, Ziegler A (2007) On the importance of equity in international climate policy: an empirical analysis. Energy Econ 29(3):545-562

Lange A, Loschel A, Vogt C, Ziegler A (2010) On the self-interested use of equity in international climate negotiations. Eur Econ Rev 54(3):359-375

Lind EA, Tyler T (1988) The social psychology of procedural justice. Plenum Press, New York

Monheim K (2015) How effective negotiation management promotes multilateral cooperation: the power of process in climate, trade, and biosafety negotiations. Routledge, London

Müller B (2001) Varieties of distributive justice in climate change. Clim Change 48(2-3):273-288

Muller H, Druckman D (2014) Justice in security negotiations. Int Negot 19(3):399-409

Niehoff BP, Moorman RH (1993) Justice as a mediator of the relationship between methods of monitoring and organizational citizen behavior. Acad Manag J 36(3):527-556

Okereke C, Dooley K (2010) Principles of justice in proposals and policy approaches to avoid deforestation: towards a post-Kyoto climate agreement. Glob Environ Change 20(1):82-95

Okereke C, Schroeder H (2009) How can justice, development and climate change mitigation be reconciled for developing countries in a post-Kyoto settlement? Clim Dev 1(1):10-15

Paterson M (2001) Principles of justice in the context of global climate change. In: Luterbacher U, Sprinz DF (eds) International relations and global climate change. MIT Press, Cambridge

Shue H (1992) The unavoidability of justice. In: Hurrell A, Kingsbury B (eds) The international politics of the environment. Oxford University Press, New York

Sjöstedt G (ed) (1993) International environmental negotiation. Sage, London

Spector BI, Zartman IW (eds) (2003) Getting it done: postagreement negotiation and international regimes. United States Institute of Peace, Washington, DC

Törnblom K, Vermunt R (2007) Towards an integration of distributive justice, procedural justice, and social resource theories. Soc Justice Res 20(3):312-335

Wagner L (2008) Problem solving and bargaining in international negotiation. Martinus Nijhoff, Leiden

Wagner L, Druckman D (2012) The role of justice in historical negotiations. Negot Confl Manag Res 5(1):49-71

Zartman IW, Kremenyuk V (eds) (2005) Peace vs. justice: negotiating forward-and backward-looking outcomes. Rowman and Littlefield, Lanham 